\section{Medieal Seiener}

pISSN 2321-7359; eISSN 2321-7367
To Cite:

Bhattacharjee A, Malhotra G, Ramteke H, Wagh D, Shinde S.

Synchronous primary malignancy of breast and endometrium in

premenopausal women - A rare case report. Medical Science, 2022, 26, ms21e1912.

doi: https://doi.org/10.54905/disssi/v26i119/ms21e1912

\section{Author Affiliation:}

${ }^{1}$ Senior Resident, Department of General Surgery, Jawaharlal Nehru medical college, Datta Meghe University of Medical Sciences, , Wardha, Maharashtra, India

${ }^{2}$ Resident, Department of General Surgery, Jawaharlal Nehru medical college, Datta Meghe University of Medical Sciences, Wardha, Maharashtra, India

3Professor, Department of General Surgery, Jawaharlal Nehru medical college, Datta Meghe University of Medical Sciences, Wardha,

Maharashtra, India

Corresponding author

Resident, Department of General Surgery, Jawaharlal Nehru medical college, Datta Meghe University of Medical Sciences,

Wardha, Maharashtra, India

Email: geetikavns@gmail.com

Peer-Review History

Received: 08 December 2021

Reviewed \& Revised: 10/December/2021 to 5/January/2022

Accepted: 6 January 2022

Published: 13 January 2022

\section{Peer-review Method}

External peer-review was done through double-blind method.

URL: https://www.discoveryjournals.org/medicalscience

\section{(ब) (1)}

This work is licensed under a Creative Commons Attribution 4.0 International License.

\section{Synchronous primary}

\author{
malignancy of breast and \\ endometrium in
}

\section{premenopausal women - A rare case report}

\author{
Anurag Bhattacharjee ${ }^{1}$, Geetika Malhotra ${ }^{2}{ }^{\circledR}$, Harshal \\ Ramteke $^{3}$, Dhirendra Wagh ${ }^{3}$, Sandip Shinde ${ }^{1}$
}

\begin{abstract}
Synchronous primary cancer in a single patient is relatively an infrequent occurrence. Combined ovarian \& endometrial cancers are well-known synchronous primary cancers in women. Although breast carcinoma and endometrial cancer are too common malignancies in the female population, they have known association in the postmenopausal phase \& not in the younger age group. Therefore, the possibility of concomitant cancer in a young woman (premenopausal) is often neglected. The current case is an example of this scenario hence presenting a very rare case of a 38 year old women diagnosed with invasive ductal carcinoma \& endometrial papillary adenocarcinoma synchronously. Since such a correlation has not been reported in the literature, the associated risk- factors are worthy of attention seeking, and the strategy for prevention is highlighted.
\end{abstract}

Keywords: Breast, Endometrium, Cancer, Multiple Primary Malignancy, synchronous or premenopausal women.

\section{INTRODUCTION}

Breast cancer is the most frequently and commonly diagnosed malignancy worldwide and is the leading cause of cancer death in women (Jemal, 2008). Endometrial cancers are the most familiar type of gynecological malignancy that is primarily seen in postmenopausal females. Association between the two conditions has long been postulated not only on the basis of the genetic correlation but also on the role of estrogen in the development of both cancers (Bani-Mostafavi, 2017). Several other mechanisms such as hereditary, immunology, environmental factors, chemicals, viruses, and chemotherapic regimens are considered pathogenic for MPM (Soliman, 2005). Tumors that are diagnosed concurrently or within 6 months are known as synchronous. With a longer period between them, the tumours are metachronous. Multiple primary malignancies (MPM) are defined as the occurrence of the primary 
malignancy with diverse histology in two or more parts of the body distinct from each other. In addition, to be indistinct, these tumors should have standard features of malignancy, and the possibility that one is the metastasis of the other must be ruled out.

We present a young premenopausal patient with two primary malignant tumours, including breast cancer (invasive-ductal Ca) and endometrial cancer (papillary adenocarcinoma), which can be regarded as synchronous MPM.

\section{CASE REPORT}

A 38yr old female referred by a gynaecologist complained of a lump in both breasts (fig 1a). Her history showed an irregular menstrual cycle of 5-7 days and an interval of 30-40 days which had been exacerbated in recent months (Heavy vaginal bleeding) despite treatment from a gynaecologist. Menarche was at 14yrs of age. Other factors like Hypertension, Diabetes, Cigarette smoking, Alcohol were unremarkable. Family history was also unremarkable. Her BMI was normal. Clinically she was grossly pale \& anaemic. On examination, a palpable hard mass of approximately size $10 \times 8 \mathrm{~cm}$ occupying all quadrants with skin involvement was noted on the right side. Left breast lump of approximate size $10 \times 6 \mathrm{~cm}$, hard in consistency with skin involvement \& pectoral muscle involvement was noted. Bilateral axillary and bilateral supraclavicular hard lymph nodes were palpable clinically staged as T4c N3b M0. On examination, a hemogram was 5gm \%. Fine needle biopsy for both lumps suggested infiltrating ductal carcinoma (grade 2), ER, PR was positive, but HER2 neu was negative. There was no distinct metastasis.

She was put on neoadjuvant chemotherapy (CAF regimen) by an oncologist after giving blood transfusions to treat her anaemia. After receiving the first cycle of chemotherapy, she further had heavy menstrual bleeding PV (DUB), for which a gynaecological opinion was taken. Transabdominal USG and D\&C were advised. USG showed the thickness of the endometrium (28mm), dilatation and curettage were planned but, given persistent heavy vaginal bleeding and low Hb, surgery (Total Abdominal Hysterectomy \& Bilateral Salpingo-oophorectomy) (fig 2) was performed after proper consultation, and the histological results showed ca endometrium (High-grade papillary adenocarcinoma) (fig 3a \& b) invading two-third of the myometrium. Post-op recovery was normal. Given high grades and two-third of myometrium involvement, she has advised radiotherapy. The patient took only two cycles of radiotherapy, and after six months, she reported back to us with the increase in the size of breast lump and appearance of satellite skin nodules (fig 1b, c) over the right breast with no distinct metastasis. Presently, the patient is on follow-up after completing 12 cycles of chemotherapy (paclitaxel regimen) and radiotherapy with partial response.

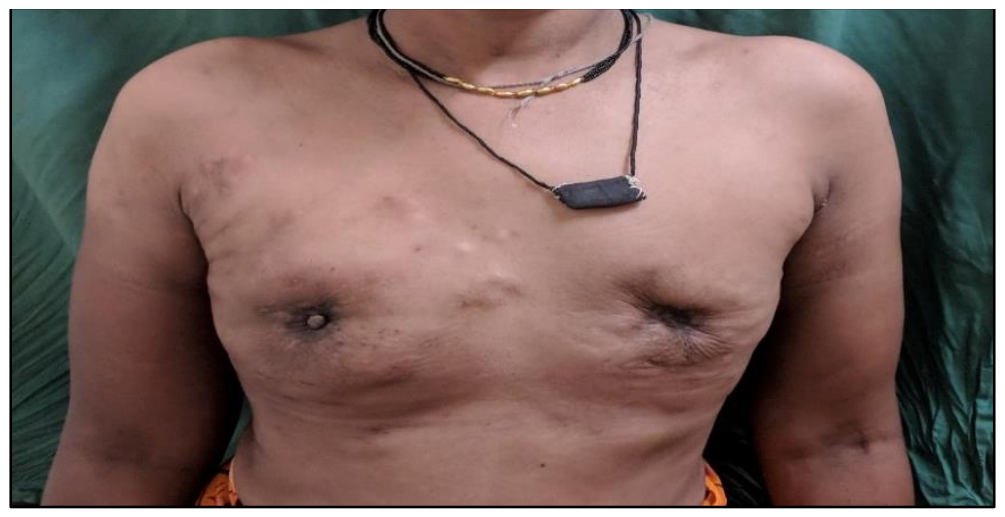

Figure 1a Invasive ductal carcinoma with bilateral breast lumps
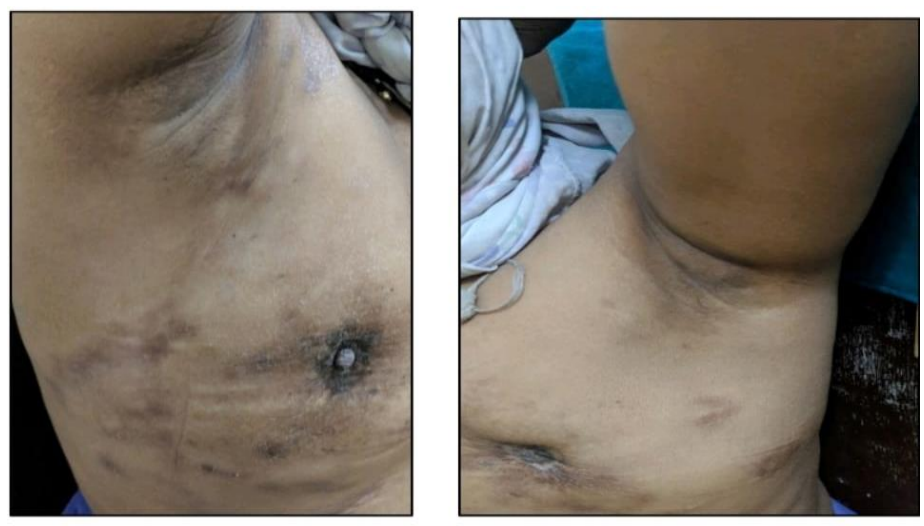

Figure 1b \& c Satellite nodules 


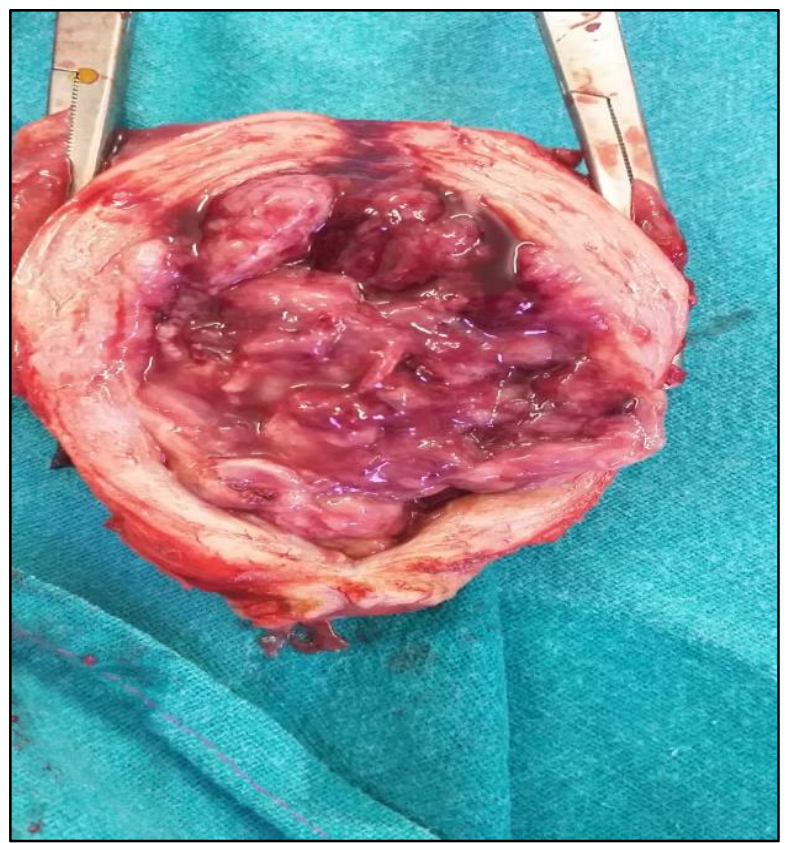

Figure 2 Total abdominal hysterectomy

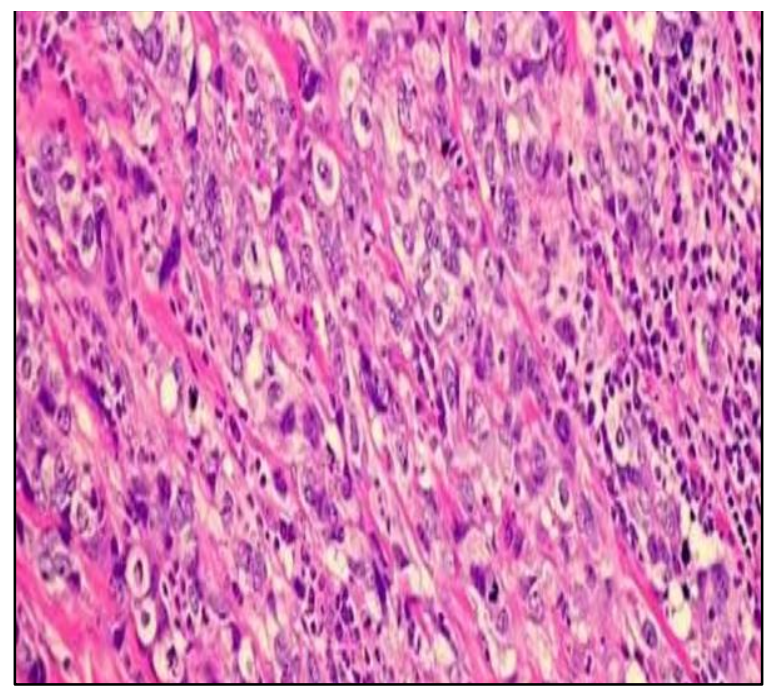

Figure 3a Invasive Ductal Carcinoma (Histopathological specimen) Grade 2

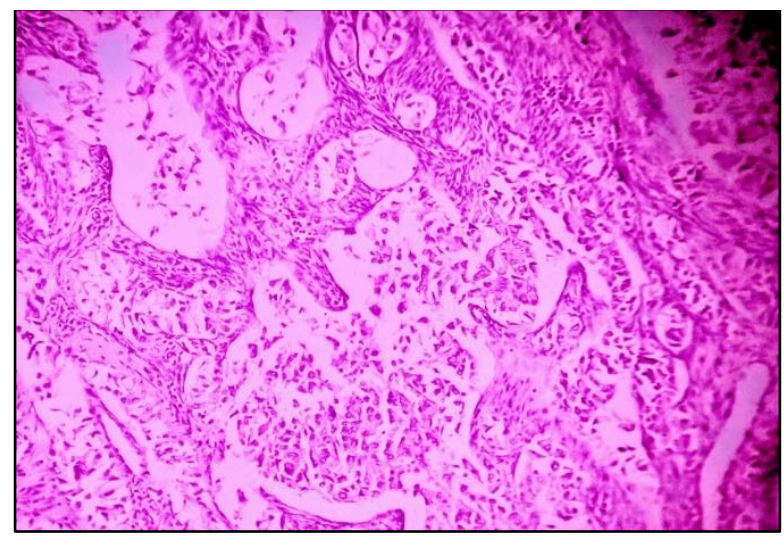

Figure 3b Ca Endometrium Adenocarcinoma (Papillary) Variant 


\section{DISCUSSION}

Breast and endometrial carcinoma are two most common malignancies in women. A potential association between Breast and endometrial cancers has been a subject of interest for many years (Yeh and Wang, 2011). But it is believed that both tumours have a similar genetic predisposition, and both are secondary to unopposed estrogen stimulation in a majority of cases (Marchesoni, 2001). In addition, the possible carcinogenic effect of tamoxifen on the endometrium in patients with breast cancer has received worldwide attention. The diagnosis of synchronous primary malignancy in one patient is relatively uncommon and sometimes very difficult to reach if the cell types are similar, or cancers of the same type are found (Mellemkjaer, 2006). In this situation, the pathological clinic criteria are most important to differentiate the two, partly because the outcome or therapeutic plan is significantly different from each other. In our case, it was easy to conclude the possibility of metastasis from one tumor to the other (EC-BC or BC-EC), as the cell types of both sites were easily distinguished.

The possibility of synchronous Endometrial \& Breast Carcinoma in one person is extremely low, as reported in one study. The diagnosis of endometrial cancer within one year after primary breast cancer is less than 0.05\% (n=525527). Additionally, a large cohort study of 188 premenopausal Endometrial carcinoma patients showed $25 \%(\mathrm{n}=48)$ with a second primary cancer, but none had synchronous Breast Carcinoma. In fact, in a research of the English language literature, the first case of synchronous Endometrial and Breast Carcinoma was reported by Tsekeris and Dimou. However, the patient was elderly (63 years). Many risk factors contribute to Endometrial \& Breast Carcinoma, and some of them are shared. The risk factors can be genetic susceptibility, hormonal, environmental, or other treatment-related factors, obesity, or the interaction of several unknown factors (Rahmani, 2018). Some of them are controversial, e.g., high BMI is shown to be directly associated with the risks of Breast Carcinoma in postmenopausal women; however, it has an opposite effect on premenopausal women. In contrast, high BMI is correlated with an increased risk of endometrial carcinoma in premenopausal and postmenopausal women. Many other factors are associated with an increase in risk of Endometrial carcinomas, such as old age, post menstrual period, nulliparous, and a positive history of irregular menstrual cycle (Colak, 2021).

Our case had an irregular menstrual history as a risk factor. Obesity, nulliparous and irregular menstrual cycle may represent less. Progesterone exposure might contribute to the development of endometrial carcinoma. In addition to many similar environmental \& hormonal factors, the exact embryological origin of the endometrium and the Breast can also be an additional associated factor 3. MPMs can generally be categorized into three major groups depending on the primary etiologic factor. The $1^{\text {st }}$ group is treatment associated with malignancies, the $2^{\text {nd }}$ group includes hereditary conditions like Cowden syndrome \& the third group is neoplasms that have common etiologic factors, genetic corealtion and the same environmental factors. According to this classification, our patient can be categorized in the $3^{\text {rd }}$ group (Arslan, 2013).

\section{CONCLUSION}

Although breast cancer \& endometrial cancer are two common cancers of women, the occurrence of both primary malignancies at the initial diagnosis is often neglected, especially in young premenopausal women. In our case, an irregular menstrual cycle was possibly the most relevant risk factor. However, all other risk factors should be taken into account by clinicians when making a decision concerning screening or strategy for prevention.

\section{Acknowledgement}

We thank the participants who were all contributed samples to the study.

\section{Author Contributions}

Dr Anurag bhattacharjee - Data acquisition, Concept and design, Guarantor

Dr Geetika Malhotra - manuscript writing and editing

Dr D.D Wagh - Critical review,

Dr Harshal Ramteke - Critical reviewer, interpretation of data

Dr Sandip Shinde - Critical reviewer

\section{Informed consent}

Written \& Oral informed consent was obtained from all individual participants included in the study. Additional informed consent was obtained from all individual participants for whom identifying information is included in this manuscript. 


\section{Funding}

The study did not receive any external funding

\section{Conflict of interests}

The authors declare that there are no conflicts of interests.

\section{Data and materials availability}

All data associated with this study are present in the paper.

\section{REFERENCES AND NOTES}

1. Arslan D, Tural D, Tatlı AM, Akar E, Uysal M, Erdoğan G. Isolated uterine metastasis of invasive ductal carcinoma. Case Rep Oncol Med 2013; 2013:793418.

2. Colak E, Erinanc OH, Ozdemir D. Diagnosis of micropapillary carcinoma of the breast by endometrial biopsy in a postmenopausal patient with abnormal uterine bleeding. Ann Ital Chir 2021; 10.

3. Jemal A, Siegel R, Ward E, Hao Y, Xu J, Murray T. Cancer Statistics 2008. CA Cancer J Clin [Internet]. 2008; 58(2):71-96.

4. Marchesoni D, Driul L, Fabiani G, Di Loreto C, Cataldi P, Mozzanega B. Endometrial histologic changes in postmenopausal breast cancer patients using tamoxifen. Int J Gynecol Obstet [Internet]. 2001; 75(3):257-62.

5. Mellemkjaer L, Friis S, Olsen JH, Scélo G, Hemminki K, Tracey E, Risk of second cancer among women with breast cancer. Int J cancer 2006; 118(9):2285-92.

6. Rahmani M, Nili F, Tabibian E. Endometrial Metastasis from Ductal Breast Carcinoma: A Case Report with Literature Review. Am J Case Rep 2018; 19:494-9.

7. Soliman PT, Oh JC, Schmeler KM, Sun CC, Slomovitz BM, Gershenson DM, Risk factors for young premenopausal women with endometrial cancer. Obstet Gynecol 2005; 105(3):575-80.

8. Yeh CC, Wang PH, Lai CR, Yen MS, Chao KC. Synchronous breast invasive ductal carcinoma and endometrial endometrioid adenocarcinoma: Case report. J Obstet Gynaecol Res [Internet]. 2011; 37(9):1246-9. 